\title{
Accessing the ANW dictionary
}

\author{
Fons Moerdijk, Carole Tiberius, Jan Niestadt \\ Institute for Dutch Lexicology (INL) \\ Leiden \\ \{moerdijk, tiberius, niestadt\}@inl.nl
}

\begin{abstract}
This paper describes the functional design of an interface for an online scholarly dictionary of contemporary standard Dutch, the ANW. One of the main innovations of the ANW is a twofold meaning description: definitions are accompanied by 'semagrams'. In this paper we focus on the strategies that are available for accessing information in the dictionary and the role semagrams play in the dictionary practice.
\end{abstract}

\section{Introduction}

In this paper we discuss the functional design of an interface for a scholarly dictionary of contemporary standard Dutch which is currently being compiled at the institute for Dutch Lexicology in Leiden. The 'Algemeen Nederlands Woordenboek' (General Dutch Dictionary), further abbreviated as ANW, has been set up as an online dictionary from the start. Thus, the ANW is not a clone of an existing printed dictionary, but it truly represents a new generation of electronic dictionaries in the sector of academic and scientific lexicography. A similar dictionary project is undertaken for German at the Institut für Deutsche Sprache in Mannheim, i.e. elexiko ${ }^{1}$.

The project runs from 2001 till 2019. We have currently finished the functional design of the interface and the first results will be published on the web in 2009.

(C) 2008. Licensed under the Creative Commons Attribution-Noncommercial-Share Alike 3.0 Unported license (http://creativecommons.org/licenses/by-nc$\underline{\mathrm{sa} / 3.0 /)}$. Some rights reserved.

\footnotetext{
${ }^{1}$ http://hypermedia.ids-

mannheim.de/pls/elexiko/p4_start.portal
}

The structure of this paper is as follows. First we will provide some background information on the ANW dictionary and we will explain what a semagram is. Then we will discuss the range of search routes that are offered to the user to exploit the information in the dictionary and we will describe the role of the semagram. The ANW dictionary is aimed at the adult Dutch language user ranging from laymen to linguists and other language professionals.

\section{The ANW dictionary}

The ANW Dictionary is a comprehensive online scholarly dictionary of contemporary standard Dutch in the Netherlands and in Flanders, the Dutch speaking part of Belgium. Object of description is the general language. Thus words that are specific to a particular region, to a particular group of people or a particular subject field are not included. The dictionary focuses on written Dutch and covers the period from 1970 till 2018. The ANW dictionary is a corpus-based dictionary based on the ANW corpus, a balanced corpus of just over 100 million words, which was compiled specifically for the project at the Institute for Dutch Lexicology. The corpus was completed in $2005^{2}$. It consists of several subcorpora: a corpus of present-day literary texts, a corpus of neologisms, a corpus of domain dependent texts and a corpus of newspaper texts. The dictionary will contain approximately 80.000 headwords with a complete description and about 250.000 smaller entries.

The ANW is a very informative dictionary. Its abstract entry structure is composed of hundreds of elements and subelements. The reason for this is that special attention is paid to words in context (combinations, collocations, idioms, prov-

\footnotetext{
${ }^{2}$ For neologisms new corpus material continues to be gathered.
} 
erbs) and to relations with other words (lexical relations like synonymy, antonymy, hyperonymy, hyponymy), to semantic relations (metaphor, metonymy, generalisation, specialisation) and to morphological patterns, the word structure of derivations and compounds. One of its main innovations is a twofold meaning description: definitions are accompanied by 'semagrams'. As semagrams play a central role in the dictionary (for understanding and production), we provide a short introduction below.

\section{The semagram}

A semagram is the representation of knowledge associated with a word in a frame of 'slots' and 'fillers'. 'Slots' are conceptual structure elements which characterise the properties and relations of the semantic class of a word meaning. On the basis of these slots specific data is stored ("fill- ers') for the word in question. In ANW jargon the abstract structure schema is called a 'type template', whereas semagram refers to such a 'type template' populated with concrete word data. Each semantic class has its own predefined type template with its own slots. For instance, the type template for the class of animals contains the slots PARTS, BEHAVIOUR, COLOUR, SOUND, BUILD, SIZE, PLACE, APPEARANCE, FUNCTION and SEX, whereas the type template for beverages has slots for INGREDIENT, PREPARATION, TASTE, COLOUR, TRANSPARANCY, USE, SMELL, SOURCE, FUNCTION, TEMPERATURE and COMPOSITION. So far we have concentrated on semagrams for nouns, those for verbs and adjectives will be different. Below we give an example of a semagram for a member of the animal class, i.e. koe (cow) (translated into English for illustration) in its meaning as a 'bovine':

\section{COW}

$\begin{array}{ll}\text { UPPER CATEGORY: } & \text { is an animal \# animal; mammal; ruminant } \\ \text { CATEGORY: } & \text { is a bovine (animal) \# bovine; ruminant } \\ \text { SOUND: } & \text { moows/lows, makes a sound that we imitate with a low, long-drawn 'boo' \# } \\ & \text { moo; low; boo } \\ & \text { is often black and white spotted, but also brown and white spotted, black, } \\ \text { COLOUR: } & \text { brown or white \# black and white; brown and white; red and white; spotted; } \\ & \text { black; blackspotted; white; brown; rusty brown } \\ & \text { is big \# big } \\ \text { SIZE: } & \text { is big-boned, bony, large-limbed in build \# big-boned, bony, large-limbed } \\ \text { BUILD: } & \text { has an udder, horns and four stomachs: paunch, reticulum, third stomach, proper } \\ \text { PARTS: } & \text { stomach \# udder; horns: paunch; rumen; honeycomb bag; reticulum; third stomach; } \\ & \text { omasum; proper stomach; abomasum } \\ \text { FUNCTION: } & \text { produces milk and (being slaughtered) meat \# milk; flesh; meat; beef; milk } \\ \text { production; meat production } \\ \text { PLACE: } & \text { is kept on a farm; is in the field and in the winter in the byre \# farm; } \\ & \text { farmhouse; field; pasture; meadow; byre; cow-house; shippon; stable } \\ \text { AGE: } & \text { is adult, has calved \# adult; calved } \\ \text { PROPERTY: } & \text { is useful and tame; is considered as a friendly, lazy, slow, dumb, curious, social } \\ \text { SEX: } & \text { animal \# tame; domesticated; friendly; lazy; slow; dumb; curious; social } \\ \text { BEHAVIOUR: } & \text { is female \# female } \\ \text { TREATMENT: } & \text { grazes and ruminates \# graze; ruminate; chew the cud } \\ \text { PRODUCT: } & \text { is milked every day; is slaughtered \# milk; slaughter } \\ \text { VALUE: } & \text { produces milk and meat \# milk; meat } \\ & \text { is useful \# useful }\end{array}$

Example 1. Semagram for koe (cow)
At present the data in the slots is completed manually by the lexicographers based on information in the ANW corpus, reference works (such as dictionaries and encyclopaedia) and their language and world knowledge. Not all slots in the type template have to be completed in all cases. Only those for which there is a value are shown in the above example. As can be seen from the semagram above, the lexicographers give the characterisation of the slots in terms of short statements about the headword. Such sentences are particularly well suited to get an impression of the meaning starting from the word form, i.e. for 'semasi- 
ological' queries. To facilitate the retrieval for queries from content or parts of the content to the matching words, the 'onomasiological queries', those sentences are complemented, after a '\#' character (a hash), with one or more keywords and possibly some synonyms or other relevant words. The data after the hash will not be visible to the dictionary user on the screen though and will only be used in searches by the computer to enhance retrieval.

A detailed description of the semagram, including its origin, motivation and the development of the type templates and their slots, can be found in Moerdijk (2008). In this paper we focus on the strategies that are available for accessing information in the dictionary and we discuss the role of the semagrams in this.

\section{Accessing the dictionary}

As was hinted at in the previous section, semagrams provide an increase and improvement in search and query facilities. This is particularly the case for queries guiding the user from content to form. For instance, a user who cannot think of e.g. the word apiarist can find this word through separate content elements (e.g. 'bees', 'keep') that he does know and can use for a search. However, with semagrams it is not only possible to go from content to the appropriate word. It is also possible to retrieve a set of words on the basis of one or more content features. Thus a user can retrieve all names for female animals in Dutch on the basis of a query combining the field CATEGORY with the value 'animal', and a field SEX with the value 'female'. In our online dictionary we wish to make all these possibilities available to the user.

Five search options are distinguished:

a) word $\rightarrow$ meaning, i.e. search for information about a word;

b) meaning $\rightarrow$ word, i.e. search for a word starting from its meaning;

c) features $\rightarrow$ words, i.e. search for words with one or more common features;

d) search for example sentences;

e) search for other dictionary information.

We believe that by presenting the search option this way (rather than using the traditional dichotomy between simple search (a) and advanced search (b, c, d, e)), users have a better overview of what they can actually search for and will be more enticed to explore the various options. Semagrams play a role in the first three search options.

\section{1 $\quad$ Word $\rightarrow$ Meaning}

This is the traditional search which allows the user to search for information about a word or phrase in the dictionary. As this is the basic search option, it is offered to the user in a central place on every page of the interface. Some form of fuzzy matching will be incorporated to take care of typing errors and incomplete input.

The ANW contains a wealth of information. To represent this to the user, we use a variation of the two-panel selector model (Tidwell 2005), where two panes are shown next to each other on the screen. (Figure 1)

The left pane contains a tree structure showing all the elements available for the lemma in question in the ANW. These tree structures look like (and work as) Windows Explorer tree structures. Advantage is that users know immediately how to deal with them. Thus the elements are hierarchically structured and can be opened and closed like in Windows Explorer. The meaning structure (the numbered elements in Figure 1) of the lemma remains visible at all times. This way the user keeps an overview and can select the information he likes to see on the right-hand-side of the screen. This is shown for the semagram of the first meaning of koe (cow) in Figure 1. The elements are presented in the same order as in the translated semagram in Example 1. ${ }^{3}$

On the article screen, the semagram is presented together with the definition. Its function is to provide, in a systemized, explicit and consistent way, more semantic and encyclopedic information than can be given in the definition. For the lemma koe (cow), for instance, it gives the user information on sound, colour and parts, which is not present in the definition.

At the bottom left of the screen, the user is given a direct link to all idioms, proverbs, example sentences and combinations for the lemma koe (cow).

\footnotetext{
${ }^{3}$ Note that the layout is still subject to change during the graphical design of the interface.
} 


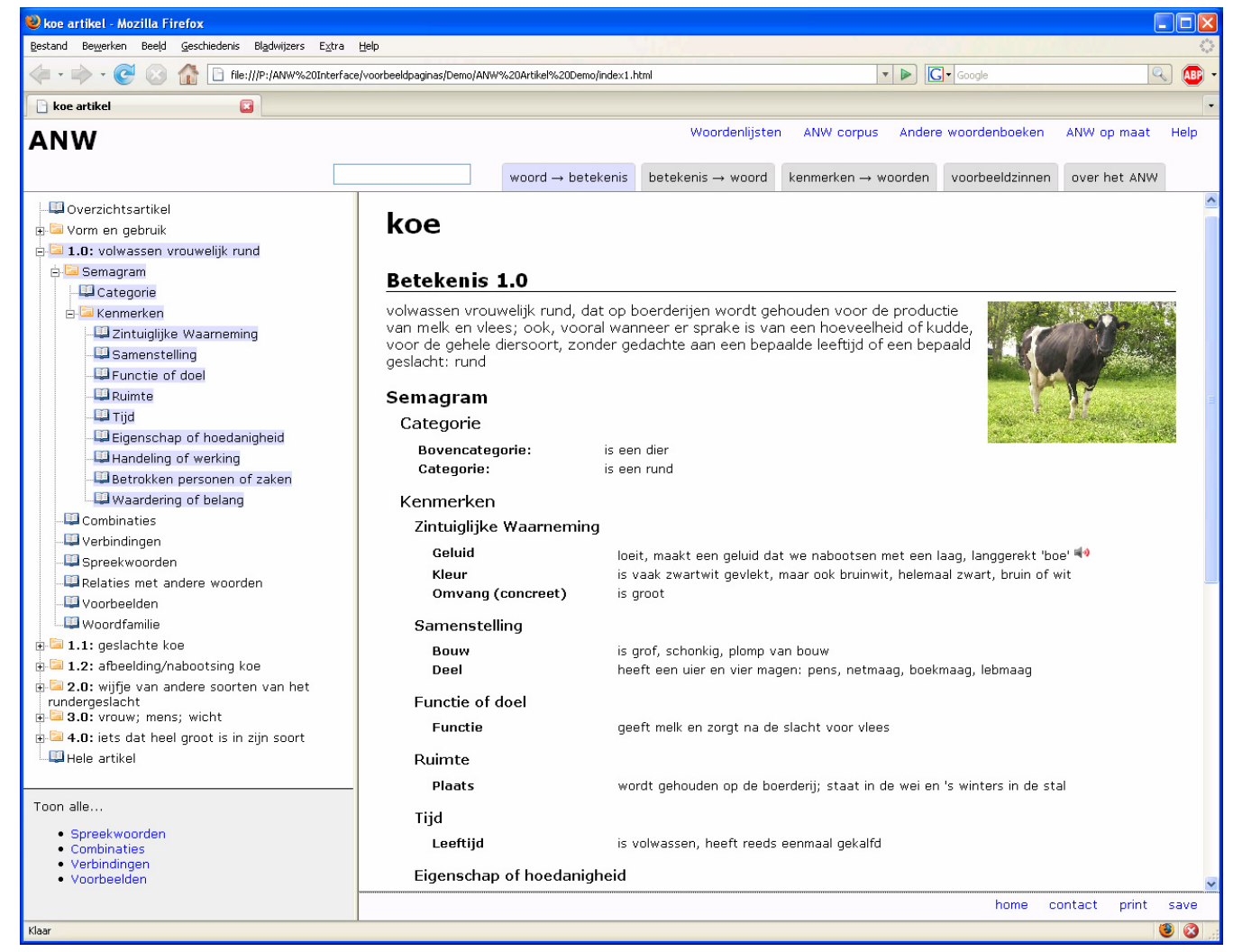

Figure 1 Article screen

\section{2 $\quad$ Meaning $\rightarrow$ Word}

By this we mean the onomasiological search where the user is looking for a word that he has forgotten or where he wants to know whether there is a word for a certain concept or not. For instance, a user may want to know whether there is a Dutch equivalent for the English cradle snatcher (i.e. one who weds, or is enamoured of, a much younger person (OED)).

Onomasiological searches in electronic dictionaries derived from printed dictionaries have not been very successful so far, mostly because such searches are primarily based on definitions. Going from a definition to a word can only succeed if the words of the user coincide (more or less) with the words in the definition, which is seldom the case (Moerdijk 2002).

As also pointed out by Sierra (2000) the ideal onomasiological search must allow writers to input the concept to be searched for through the ideas they may have, using words in any order. The system must be so constructed that it accepts a wide range of words which it then analyses in order to point the user to the word that most closely approaches the concept he had in mind when he started the search.

Recent work in computational linguistics has therefore looked at the possibility of using associative networks (Zock \& Bilac 2004) or a combination of definitions and a resource such as WordNet (El-Kahlout \& Oflazer 2004).

It is obvious that the information in the semagrams plays an essential role in the success of onomasiological queries in the ANW. However, rather than just accepting a wide range of words as input, we believe that the format in which the input query is obtained can also help to increase the success rate.

Therefore, we offer the user two alternatives for onomasiological queries. First, the user can search by giving a definition, a description, a paraphrase or by summing up synonyms or other words that he can associate with the word he is looking for. This input will be subject to some linguistic analysis including stemming and removal of stop words. Second, there is a guided search based on the semagram. The user is asked to choose a category (the semantic class or subclass) from a menu (is it a thing, a person, an animal, a vehicle, etc.?). This is a subset of the total number of semantic classes that are distinguished in the ANW. Once the user has selected a category, 
the feature slots of the type template for that category appear on the screen and the user is asked to fill in the value(s) that spring to mind. Again we do not present the full list of feature slots of the type template of that particular semantic class, but rather a dozen or so (which have been automatically deduced on the basis of completed semagrams), as we do not want to put off the user with excessively long lists which he needs to complete before he gets an answer. We illustrate this with an example for animals.

Assume the user is looking for the name of a particular breed of dogs, e.g. borzoi (barzoi in Dutch), but cannot remember the word. In order to find the answer, he selects the category 'animal' from the menu. He is then presented with a list of features that are characteristic for animals (Figure 2). He completes the most prominent ones for the animal he is thinking of e.g. BEHAVIOUR: quiet, intelligent and independent; SOUND: barks; CLASS: greyhound; PLACE: Russia; SIZE: large; BUILD: strong and graceful; APPEARANCE: long-haired; MOVEMENT: sprinter.

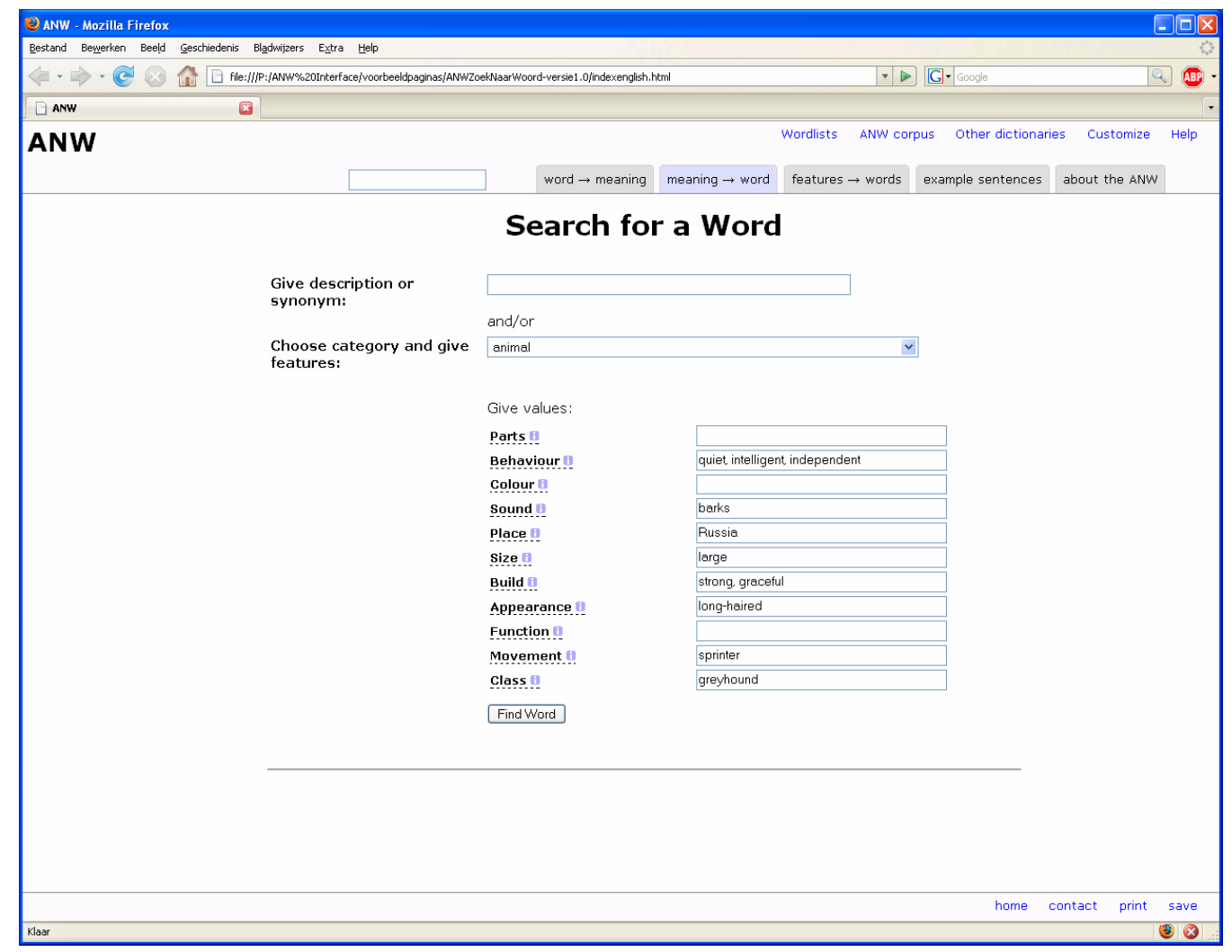

Figure 2 Screenshot Meaning $\rightarrow$ Word

The input from the user is then compared to the data in the dictionary database (semagrams, definitions, lexical relations and 'contextants $\left.{ }^{4}\right)$. Now the words behind the hashes are also involved in the retrieval process and the matching cases (in the best scenario just one!) are shown. It is not necessary that the feature-

\footnotetext{
${ }^{4}$ We define 'contextants' as words which do not occur in direct combination with the headword, but do occur in a wider context and are semantically relevant for the headword. This is a separate information category in the microstructure of the ANW.
}

value combinations match exactly one-to-one. For instance, in our example, one of the values given for BEHAVIOUR, i.e. intelligent, matches the value for PROPERTY in the semagram for barzoi (borzoi).

The results are then presented in a list, ordered by relevance. Each result is accompanied by a 'mini definition' 5 such that the user can immediately see which word (sense) he is looking for.

\footnotetext{
${ }^{5}$ A shortened version of the definition.
} 


\section{3 $\quad$ Features $\rightarrow$ words}

This option is particularly relevant for linguists and other language professionals. It enables them to gather words that share one or more identical features within the main dimensions of the ANW, i.e. orthography, pronunciation, morphology, pragmatics, meaning, combinatorics, idioms, etymology. The semagram is of course active in searches in the semantic domain. Its role is to some extent comparable to its role in the search for a word, going from content to form, but users can now search for all the words that belong to a certain semantic class, for all the words that share one or more particular features, or for all the words sharing both class and certain features, instead of searching for a particular word to express a concept. Here the user is presented the full list of feature slots that occur in one or more of the predefined type templates. This means a total of nearly 200 features can be searched for.
To assist the user in finding his way through this forest of criteria, they are presented in a structured way much like the tree structure which is used for navigation on the article screen. We illustrate this with an example query in Figure 3. The user starts from an empty query screen. He is asked to select criteria from the tree structure on the left. By default, the user searches for words, but he can also search for proverbs or idioms which will result in a different feature tree as only a subset of the criteria that can occur in a query for words apply to idioms and proverbs. In our example the user wants to find all words for long-haired animals (semagram) which consist of two syllables and have alternating stress (orthography and pronunciation). Again barzoi (borzoi) will be among the results.

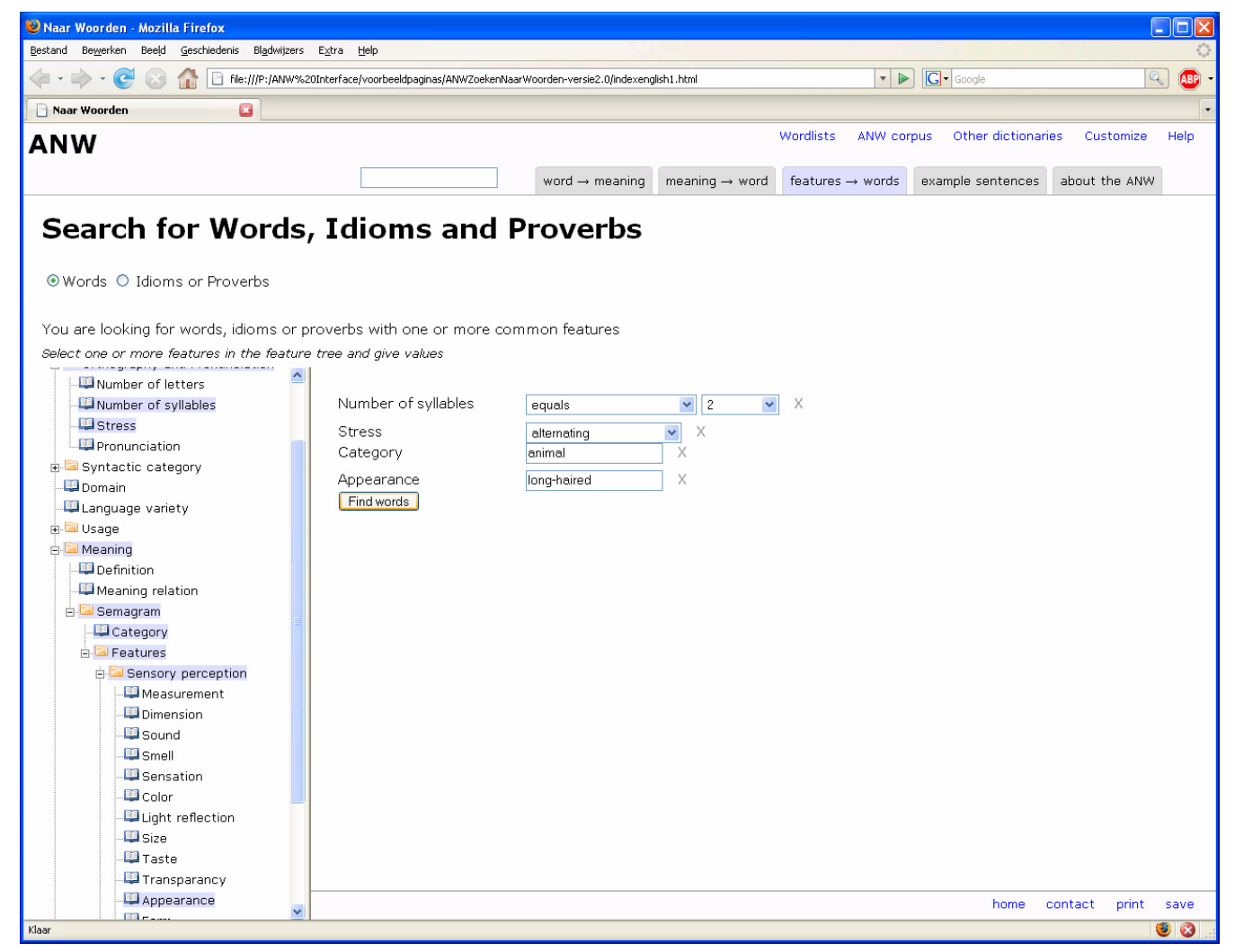

Figure 3 Screenshot Features $\rightarrow$ Words

This search option can also be used to resolve the so-called tip-of-the-tongue problems where a user is looking for a word which he cannot access in his memory, but where he does know, for instance, what the word looks like (e.g. its beginning, number of syllables) and its part of speech.
For example, a user who is unsure whether the particular breed of dogs he is looking for should be called barzoi or borzoi in Dutch, can find the answer by specifying that the form ends in $-z o i$, the word consist of two syllables, that it is a noun 
and that it refers to a breed of dogs (animal category) with long hairs (appearance).

Obviously users will be offered the possibility to save their queries in a kind of 'search templates' to avoid having to reconstruct the same query over and over again.

\subsection{Search for examples}

This option allows the user to search for example sentences based on a set of 5 criteria, i.e. word(s), author, source, domain and date. For instance, a user could search for all example sentences with the words koe (cow) and schaap (sheep) in the period from 2000 - 2002 (date). No combo boxes are used for author and source. Although we do not reckon that the user knows which authors and sources are cited in the dictionary, the lists would be excessively long and we assume that the user will only use these criteria in a search to see which other examples are available from a particular author or source he has retrieved in a previous query. Users will also be offered the possibility to link through to more examples of the same source or author by clicking on a particular source or author on the results page.

\subsection{Search for information about the ANW}

The final search option groups primarily dictionary specific queries and queries of an administrative nature, much like a Frequently Asked Questions page. Here the user will find queries about frequency such as how many lemmas are dedicated to lexicalised phrases? How many names are there in the dictionary? How many nouns? How many semagrams? How many Flemish words? It also comprises questions such as what kind of dictionary is the ANW? How big is the ANW corpus? Which images are included?

\section{Conclusion}

In this paper we have discussed the functional design of an interface for an electronic dictionary of Dutch, the ANW. We have focused on the access strategies that are offered and the role semagrams play in this. We have shown that semagrams provide an increase in search and query facilities. One the one hand, they lead to a much richer and more consistent semantic description in 'semasiological' queries. On the other hand, they are particularly well-suited to support 'onomasiological' queries by offering a structured way to find words through separate content elements.

\section{References}

El-Kahlout, İlknur Durgar \& Kemal Oflazer. 2004. Use of Wordnet for Retrieving Words from their Meanings. In Proceedings of the Global Wordnet Conference (GWC2004). 118-123.

Moerdijk, Fons. 2002. Het woord als doelwit. Amsterdam, Amsterdam University Press.

Moerdijk, Fons. 2008. Frames and semagrams; Meaning description in the General Dutch Dictionary. In Proceedings of the Thirteenth Euralex International Congress, EURALEX 2008. Barcelona.

Sierra, Gerardo. 2000. The onomasiological dictionary: a gap in lexicography. In Proceedings of the Ninth Euralex International Congress, EURALEX 2000 I, 223-235. Institut für Maschinelle Sprachverarbeitung, Universität Stuttgart.

Tidwell Jenifer. 2005. Designing Interfaces. O’Reilly.

Zock, Michael \& Slaven Bilac. 2004. Word lookup on the basis of association: from an idea to a roadmap. In Proceedings of the Workshop on Enhancing and using electronic dictionaries, COLING 2004. 2935 . 